\title{
Serotonergic Modulation of Synapses in the Developing Gerbil Lateral Superior Olive
}

\author{
KENT K. FITZGERALD ${ }^{1}$ AND DAN H. SANES ${ }^{1,2}$ \\ ${ }^{1}$ Center for Neural Science and ${ }^{2}$ Department of Biology, New York University, New York City, New York 10003
}

Fitzgerald, Kent K. and Dan H. Sanes. Serotonergic modulation of synapses in the developing gerbil lateral superior olive. J. Neurophysiol. 81: 2743-2752, 1999. The lateral superior olive (LSO) is a primary site of binaural convergence that responds selectively to changes in interaural level difference (ILD) by integrating ipsilateral excitatory and contralateral inhibitory inputs. The circuit matures during the first three postnatal weeks, undergoing several structural and functional changes that are influenced by afferent activity. Therefore modulation of synaptic activity by neuromodulators may participate in the maturation of this circuit. The present study describes robust effects of serotonin $(5-\mathrm{HT})$ on LSO synaptic function. Using whole cell voltage-clamp recording from gerbil LSO neurons (postnatal days 6-13) in an in vitro slice preparation, we have identified several distinct forms of serotonergic modulation of spontaneous and evoked synaptic transmission. First, 1-2 min application of 5-HT (100 $\mu \mathrm{M})$ activated prolonged bursts of spontaneous inhibitory postsynaptic currents (IPSCs). However, there was an age-dependent decline, such that this effect rarely was observed beyond postnatal day 8. 5-HT apparently increased the excitability of inhibitory afferents, because 5-HT-induced IPSCs were blocked by tetrodotoxin. A second effect of 5-HT was to depress rapidly and profoundly the amplitude of electrically evoked excitatory postsynaptic currents (EPSCs). In contrast, 5-HT also depressed evoked IPSCs but to a significantly lesser degree. The receptor subtypes mediating these effects were examined using specific 5-HT agonists and antagonists. A 5- $\mathrm{HT}_{1}$ agonist, 5-carboxamidotryptamine, produced EPSC depression but did not induce spontaneous IPSCs. A 5- $\mathrm{HT}_{2}$ agonist, $\alpha-\mathrm{Me}-5-\mathrm{HT}$, reproduced all the observed effects of 5-HT (PSC depression as well as induction of spontaneous IPSCs), whereas a 5- $\mathrm{HT}_{2}$ antagonist, ketanserin, blocked the induction of spontaneous IPSCs. Therefore induction of spontaneous IPSCs is mediated by $5-\mathrm{HT}_{2}$ receptors, whereas both $5-\mathrm{HT}_{1}$ and $5-\mathrm{HT}_{2}$ receptor types contribute to PSC depression. Serotonergic modulation of LSO synapses may have consequences for both developmental plasticity and auditory function. Serotonergic induction of IPSCs was observed primarily in young animals and thus may represent a mechanism for amplifying the activity of inhibitory synapses in LSO during a period of use-dependent plasticity in postnatal development. PSC depression, which preferentially affects excitation, is a potential mechanism for modulation of ILD tuning.

\section{N T R O D U C T I O N}

The lateral superior olive (LSO) is a primary site of binaural convergence in the mammalian auditory system, receiving ipsilateral excitatory input from the anteroventral cochlear nucleus (AVCN) (Cant and Casseday 1986; Warr 1982) and contralateral glycinergic inhibition from the medial nucleus of the trapezoid body (MNTB) (Glendenning et al. 1985; Moore

\footnotetext{
The costs of publication of this article were defrayed in part by the payment of page charges. The article must therefore be hereby marked "advertisement" in accordance with 18 U.S.C. Section 1734 solely to indicate this fact.
}

and Caspary 1983; Rasmussen 1946; Sanes 1990; Sommer et al. 1993; Spangler et al. 1985; Wu and Kelly 1992). By integrating these opposing bilateral inputs, LSO neurons produce an output that varies with interaural level difference (ILD), a principal cue for sound localization in the horizontal plane (Boudreau and Tsuchitani 1970; Caird and Klinke 1983; Galambos et al. 1959; Sanes and Rubel 1988). The function of LSO clearly depends on an appropriate balance of excitatory and inhibitory synaptic strength, raising the interesting question of how suitable synaptic strengths are established during development and regulated in the adult.

During early postnatal life, the development of LSO synapses is characterized by refinement of both pre- and postsynaptic morphology and changes in the efficacy of synaptic transmission (Kotak and Sanes 1996; Sanes 1993; Sanes and Siverls 1991; Sanes et al. 1992a). These developmental changes are, at least in part, activity-dependent, since manipulations that alter levels of inhibitory synaptic transmission interfere with normal development (Aponte et al. 1996; Kotak and Sanes 1996; Kotak et al. 1998; Sanes and Chokshi 1992; Sanes and Takacs 1993; Sanes et al. 1992a). These manipulations are effective before the onset of sensitivity to airborne sound at postnatal day (P) 12, suggesting that spontaneous activity, generated either in the cochlea or centrally, is important for normal development. Therefore neuromodulation of spontaneous activity levels could play a critical developmental role.

One candidate modulator of activity in developing LSO synapses is the monoamine, serotonin (5-HT). 5-HT is well characterized throughout the CNS and is one of several neuromodulators that exert influences on neural development (Bear and Singer 1986; Kasamatsu and Pettigrew 1976; Lauder 1988; Whitaker-Azmitia et al. 1996). 5-HT and its receptors are present in brain stem and midbrain auditory nuclei, including cochlear nucleus, superior olivary complex, and inferior colliculus (Harvey et al. 1993; Klepper and Herbert 1991; Thompson et al. 1994, 1995). Furthermore 5-HT has been shown to play a role in postnatal developmental plasticity in several brain areas, including the organization of thalamocortical afferents in somatosensory cortex and the formation of ocular dominance columns in visual cortex (Bennett-Clarke et al. 1994; Gu and Singer 1995). Therefore the first aim of this study was to determine whether 5-HT produces modulation of spontaneous synaptic activity in the gerbil LSO that could contribute to developmental plasticity.

The second motivation for this study was to explore forms of serotonergic modulation that may have implications for auditory processing. Because the ILD sensitivity of LSO neurons 
depends on the balance of excitatory and inhibitory synaptic inputs, differential modulation of these synaptic strengths could modify or regulate ILD processing. Although serotonergic innervation is present throughout LSO and its input nuclei, the effects of 5-HT on stimulus-evoked synaptic responses in LSO are unknown. To approach this issue, we examined the ability of 5-HT to modulate electrically evoked excitatory and inhibitory postsynaptic currents (EPSCs and IPSCs) in LSO. These forms of modulation presumably reflect the effects of 5-HT on acoustically evoked stimuli in the intact nervous system.

Some of these results have been presented in abstract form (Fitzgerald and Sanes 1996, 1997).

\section{METHODS}

Whole cell patch-clamp recordings were performed in acute brain stem slices from gerbils (Meriones unguiculatus), age P6-13. All procedures were reviewed and approved by the New York University Institutional Animal Care and Use Committee. Gerbils were anesthetized with chloral hydrate $(400-500 \mathrm{mg} / \mathrm{kg}$ ip) and decapitated, the brain stem and midbrain were rapidly removed, and 300- to $400-\mu \mathrm{m}$ transverse sections containing the superior olivary complex were cut on a vibratome. After sectioning, slices were incubated at room temperature for $1-2 \mathrm{~h}$ in oxygenated artificial cerebrospinal fluid (ACSF) consisting of (in mM) $123 \mathrm{NaCl}, 4 \mathrm{KCl}, 1.2 \mathrm{KH}_{2} \mathrm{PO}_{4}, 1.3$ $\mathrm{MgSO}_{4}, 28 \mathrm{NaHCO}_{3}, 15$ glucose, $2.4 \mathrm{CaCl} 2$, and $0.4 \mathrm{~L}$-ascorbic acid, continuously bubbled with $95 \% \mathrm{O}_{2}-5 \% \mathrm{CO}_{2}$. During recording, slices were superfused with ACSF at room temperature. To directly activate the synaptic inputs to LSO, bipolar stimulating electrodes were positioned over the ipsilateral afferents from the cochlear nucleus and over the ipsilateral MNTB. In some experiments, a stimulating electrode was placed at the midline, thereby activating contralateral cochlear nucleus afferents and synaptically activating MNTB neurons. Brief $(0.1 \mathrm{~ms})$ constant voltage isolated pulses $(1-100 \mathrm{~V})$ were applied to activate afferents.

LSO was identified visually, and pipettes of 5-8 M $\Omega$ resistance were used to obtain voltage-clamp recordings in the whole cell configuration, using the blind-patch technique. A few cells were filled with biocytin for subsequent verification of their location. The internal pipette solution consisted of (in mM) 127.5 Cs gluconate, 0.6 EGTA, 10 HEPES, 2 $\mathrm{MgCl}_{2}, 5 \mathrm{KCl}, 2 \mathrm{ATP}, 0.3 \mathrm{GTP}$, and $5 \mathrm{~N}$-(2,6-dimethylphenylcarbamoylmethyl)triethylammonium bromide (QX-314, Alomone Labs; $\mathrm{pH}=7.2$ ). Series resistance was compensated $\sim 60 \%$. A measured liquid junction potential of $8 \mathrm{mV}$ was not corrected. For each neuron recorded, synaptic inputs were characterized by individually activating the ipsilateral and contralateral (i.e., MNTB) afferents. PSC waveforms were digitized at a sampling rate of $10 \mathrm{kHz}$ and stored for subsequent analysis using custom software. In experiments examining spontaneous PSCs, $\mathrm{I}_{\mathrm{m}}$ was sampled continuously for 5-10 min (Axotape, Axon Instruments) and saved for subsequent analysis.

To assess the effects of 5-HT and 5-HT agonists on evoked EPSC and IPSC amplitudes, holding potential $\left(V_{\mathrm{H}}\right)$ in the range $-40-0 \mathrm{mV}$ was selected such that stimulation of ipsilateral cochlear nucleus afferents produced a net inward PSC, and MNTB stimulation produced a net outward PSC. In a few experiments, a more negative $V_{\mathrm{H}}$ $(-55$ to $-80 \mathrm{mV})$ was used, but IPSCs were not analyzed in these experiments. Ipsilateral and contralateral afferents were activated alternately at 10 - to 40 -s intervals at a stimulus level that elicited a PSC amplitude of $50-100 \%$ of maximum.

After a baseline period of $90-120 \mathrm{~s}$, the drug of interest was bath-applied through the superfusion system for a period of 60-120 s. In most cases, only one drug was tested in each slice; when multiple drugs were used, $\geq 30$ min was allowed for recovery between trials. When more than one concentration of a drug was tested in a single slice, lower concentrations were used first to minimize desensitization effects. Agonists and antagonists included: 5-HT creatinine sulfate, 5-carboxamidotryptamine maleate, $\alpha$-Me-5-hydroxytryptamine maleate, and ketanserin tartrate (Research Biochemicals International); strychnine hydrochloride, (-)-bicuculline methobromide, and tetrodotoxin (Sigma); and kynurenic acid (Fluka). The frequency of spontaneously occurring PSCs was counted manually during playback of the digitized $\mathrm{I}_{\mathrm{m}}$ records. For quantitative comparisons, spontaneous PSC frequency and average amplitude of evoked PSCs were determined during a 1-min baseline period before drug application, and a 1 -min test period beginning 1 min after the onset of drug application. Spontaneous PSCs were defined as those that occurred outside of a 500 -ms period after afferent stimulation. Changes in PSC frequency or amplitude were assessed using a $t$-test for related means to compare baseline and test periods. Comparisons between age groups were made by analysis of variance (ANOVA). All probability values are two-tailed. Data presented are means $\pm \mathrm{SE}$.

\section{RES U L T S}

Most LSO neurons recorded were in the high-frequency (medial) limb of LSO and most received ipsilateral synaptic excitation and contralateral (MNTB-evoked) inhibition. Synaptic currents that were atypical (e.g., ipsilateral inhibition) or that contained mixed inward and outward components that could not be visually resolved were not included in the analysis. Baseline evoked PSC amplitudes ranged from 19 to 715 pA (EPSCs) and 16 to 560 pA (IPSCs). Evoked PSCs, particularly MNTB IPSCs, often consisted of multiple summating components. Synaptic amplitudes did not exhibit decrement when repeatedly activated at frequencies of $0.025-0.1 \mathrm{~Hz}$.

\section{Serotonergic induction of spontaneous IPSCs in LSO}

5-HT produced a striking and prolonged increase in the frequency of spontaneous outward PSCs in many LSO neurons (Fig. 1). Because $V_{\mathrm{H}}$ was chosen so that evoked ipsilateral EPSCs were inward and evoked contralateral IPSCs were outward in polarity, spontaneously occurring outward currents were interpreted as IPSCs. Spontaneous IPSCs were typically $10-50 \mathrm{pA}$ in amplitude and appeared to be unitary synaptic currents. Before 5-HT application, most LSO neurons (78\%) had low rates of spontaneous IPSCs ( $\leq 10$ PSCs/min), whereas a few cells had high rates of spontaneous IPSCs, up to 164 IPSCs/min. 5-HT $(100 \mu \mathrm{M})$ induced an increase (defined as a change $>10$ IPSCs/min) in the frequency of spontaneous PSCs in 28 of 55 cells examined (51\%). Although this effect was variable in magnitude and was absent in half the cells, the mean increase in frequency of spontaneous IPSCs in all 55 cells was highly significant (Fig. 1C). There was no correlation between the baseline rate of spontaneous IPSCs and the magnitude of the increase produced by 5-HT. The latency of onset of spontaneous IPSCs was also variable; the example in Fig. $1 B$ shows a relatively long latency $(\sim 1 \mathrm{~min})$, while in other cells onset occurred in $<10 \mathrm{~s}$, presumably reflecting the depth of the cell in the slice and access to the ACSF.

Several manipulations were performed to explore the ionic and pharmacological nature of the spontaneous PSCs induced by 5-HT. To confirm that outward PSCs were inhibitory, several experiments were conducted in the presence of $5 \mathrm{mM}$ kynurenic acid to block glutamatergic excitatory transmission. Five of six cells recorded in kynurenic acid exhibited substantial induction of spontaneous IPSCs by 5-HT (mean increase 


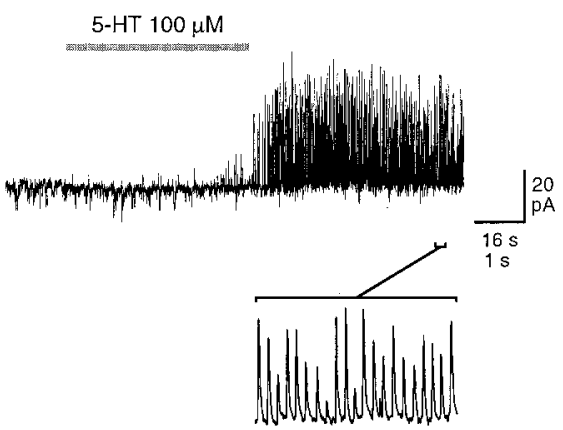

C

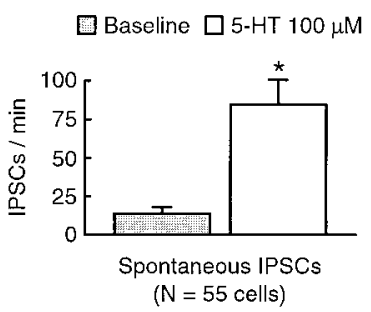

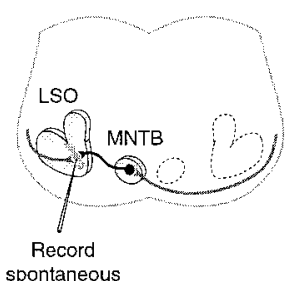

$$
\text { spontaneous }
$$

D

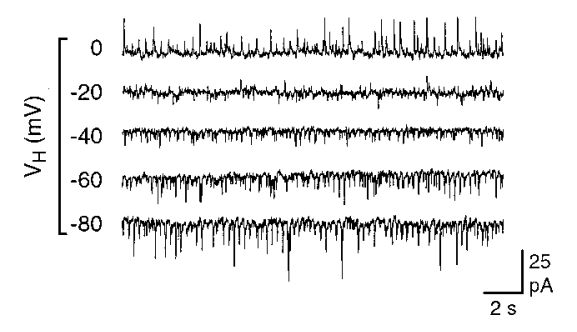

FIG. 1. Induction of spontaneous inhibitory postsynaptic currents (IPSCs). A: schematic of the brain stem slice preparation in which lateral superior olive (LSO) recordings were done. $B$ : voltage-clamp recording from a P6 LSO neuron demonstrates the induction of spontaneous IPSCs by serotonin (5-HT; bar). $V_{\mathrm{H}}=-15 \mathrm{mV}$. Evoked excitatory postsynaptic currents (EPSCs) have been deleted from the trace for clarity. In this example, no spontaneous IPSCs were observed before the application of 5-HT. Onset of spontaneous activity followed drug application by $\sim 60 \mathrm{~s}$. $C$ : summary data (mean $\pm \mathrm{SE}$ ) from 55 LSO neurons. Spontaneous IPSCs were counted in 1-min periods before and after 5-HT application. 5-HT produced a significant increase in the rate of IPSCs $(* P<0.0005) D$ : reversal potential of spontaneous IPSCs. Successive voltage-clamp traces from a P7 LSO neuron, showing spontaneous IPSCs after 5-HT $(100 \mu \mathrm{M})$ application. To determine the synaptic reversal potential, $V_{\mathrm{H}}$ was varied through the range of 0 to $-80 \mathrm{mV}$ as indicated (traces are offset for clarity). Spontaneous IPSCs reversed polarity at $V_{\mathrm{H}}=-20 \mathrm{mV}$.
$217 \pm 127$ PSCs/min). Figure $1 D$ shows an experiment in which the reversal potential of IPSCs was determined by first applying 5-HT and then varying $V_{\mathrm{H}}$ through the range 0 to -80 $\mathrm{mV}$. IPSCS in this example reversed at approximately -20 $\mathrm{mV}$, a level that is typical for the reversal potential of MNTBevoked inhibitory synapses in gerbil LSO neurons at this age (Kotak et al. 1998). In another experiment, the frequency and amplitude of 5-HT-induced spontaneous IPSCs in LSO were attenuated by the glycine antagonist strychnine (Fig. 2), indicating that the spontaneous IPSCs were partially glycinergic. This result is consistent with the recent finding that at MNTBevoked inhibitory synaptic currents at P8-11 consist of approximately equal contributions of glycine and GABA (Kotak et al. 1998). In addition, four cells (P8) were recorded in the presence of $5 \mu \mathrm{M}$ strychnine plus $20 \mu \mathrm{M}$ bicuculline to isolate EPSCs (see following text). None of these cells displayed increased spontaneous PSCs after application of 5-HT.

Spontaneous IPSCs recorded in LSO could arise from two possible mechanisms: spontaneous action potential firing in presynaptic neurons or an increase in non-spike-dependent release of transmitter. To distinguish between these possibilities, we examined whether spontaneous IPSCs induced by 5-HT were blocked by the $\mathrm{Na}^{+}$channel blocker, tetrodotoxin (TTX). In three neurons, after the induction of spontaneous IPSCs by 5-HT (100 $\mu \mathrm{M})$, TTX $(1 \mu \mathrm{M})$ was applied. In all cases, spontaneous IPSCs were blocked by TTX (Fig. 3). Thus induction of spontaneous IPSCs by 5 -HT requires $\mathrm{Na}^{+}$-dependent action potentials.

\section{Age dependence of induction of spontaneous IPSCS}

Induction of spontaneous IPSC was examined in cells from P6-13 animals and was observed predominantly in cells from younger animals. 5-HT produced significant increases in the frequency of spontaneous IPSCs in neurons from P7 to 8 day animals but not at higher ages (Fig. 4). Furthermore, a linear- model one-way ANOVA revealed a significant effect of age on the change in spontaneous IPSC frequency $\left(\mathrm{F}_{1,53}=4.79, P<\right.$ $0.05)$. This decline in effect with age resulted from a decrease in the proportion of cells that were responsive to 5-HT: 24 of 39 cells $(62 \%)$ from P6 to 8 animals exhibited increased spontaneous activity (change $>10$ IPSCs/min), whereas only 4 of 16 cells (25\%) from P9 to 13 animals showed increases in spontaneous IPSCs. The age dependence of this form of modulation suggests that it may have a specific function during postnatal development.

\section{Serotonergic modulation of evoked PSCs in LSO neurons}

To determine the effects of 5-HT on stimulus-evoked synaptic transmission in LSO, ipsilateral EPSCs and contralateral IPSCs were elicited with electrical stimulation at 10- to 40-s intervals, and 5-HT was bath-applied for 1-2 min. 5-HT generally exerted depressive effects on evoked PSCs. In the example shown in Fig. 5B, $100 \mu \mathrm{M} 5$-HT produced rapid and pronounced depression of EPSC amplitude, which lasted for the remainder of the 10 min recording period. Evoked IPSCs, recorded in another cell, were only slightly depressed (Fig. 5C).

Results from a number of such experiments are shown in Fig. 5, $D-F$. Application of $10 \mu \mathrm{M}$ 5-HT produced significant depression of EPSC amplitude but did not affect IPSCs (Fig. 5D). 5-HT (100 $\mu \mathrm{M})$ produced more substantial EPSC depression and also significantly depressed IPSC amplitude (Fig. 5E). The mean depression of EPSCs by $100 \mu \mathrm{M} 5$-HT was $66 \pm$ $5 \%$; several cells exhibited almost complete $(\leq 90 \%)$ depression. Depression was long lasting (typically 5-10 min), and EPSC amplitudes rarely recovered completely to baseline levels. To assess the relative effect of 5-HT on excitation and inhibition, PSC depression was expressed as a percentage of the baseline PSC amplitude, and the percent reduction of EPSCs and IPSCS was compared (Fig. 5F). Serotonergic depression of excitation was significantly greater than depression 
A
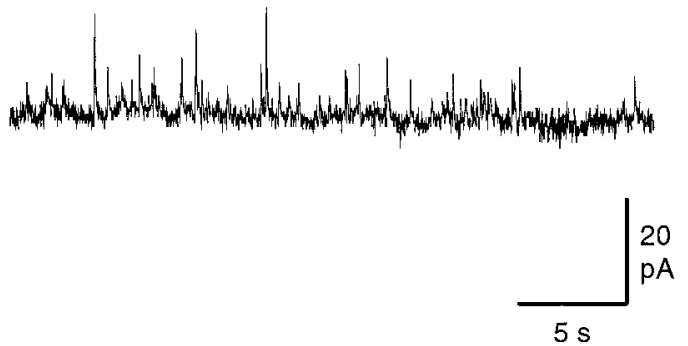

B
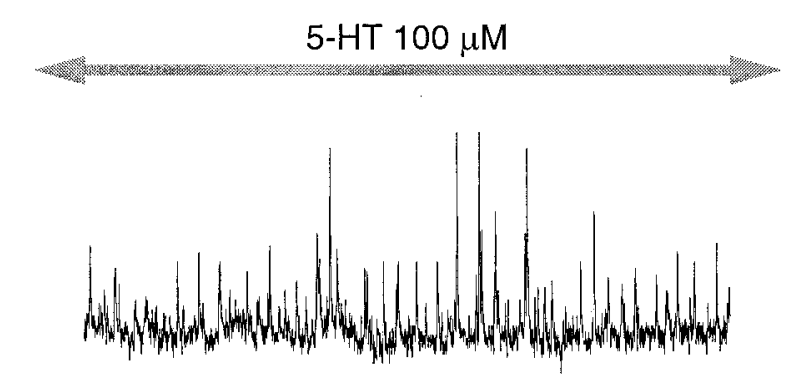

C
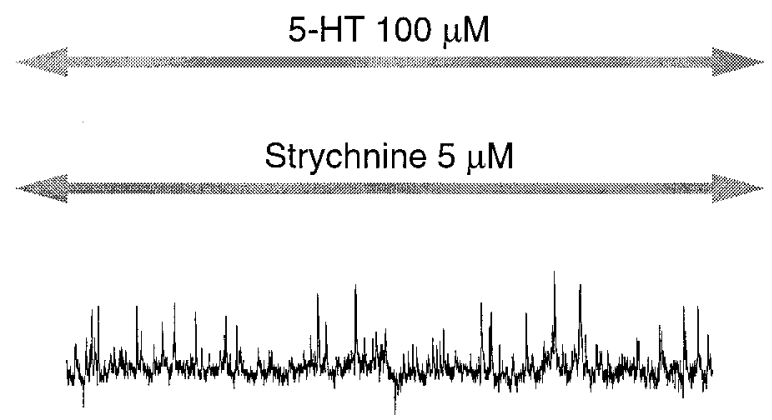

FIG. 2. Reduction of spontaneous IPSCs by strychnine. Voltage-clamp recordings from a P8 LSO neuron. $V_{\mathrm{H}}=0 \mathrm{mV}$. A: low rate of IPSCs was seen before 5-HT. B: 5-HT application induced frequent spontaneous IPSCs (trace shown is $60 \mathrm{~s}$ after 5 -HT application). $C: 5 \mu \mathrm{M}$ strychnine subsequently was applied. Strychnine reduced both the frequency and amplitude of spontaneous IPSCs. Elapsed time between traces is $90 \mathrm{~s}$.

of inhibition. There was no relationship between age and the modulation of evoked PSCs. Several additional experiments were conducted to examine pharmacologically isolated EPSCs and IPSCs. Evoked EPSCs recorded in $5 \mu \mathrm{M}$ strychnine plus $20 \mu \mathrm{M}$ bicuculline were reduced by 5-HT in four of four cells (mean decrease $53 \pm 17 \%$ ). Evoked IPSCs isolated with $5 \mathrm{mM}$ kynurenic acid were not significantly reduced by 5 -HT $(n=6)$.

\section{Pharmacological specificity of serotonergic modulation}

In the preceding experiments, 5-HT exerted distinct actions on spontaneous IPSCs and evoked EPSCs and IPSCs in LSO. These heterogeneous forms of modulation may result from independent mechanisms and may be mediated by different receptor subtypes. To determine the 5-HT receptor types involved, we assessed the ability of 5-HT agonists and antagonists to reproduce or block the effects of 5-HT on LSO neurons.

The 5-HT 1 agonist, 5-CT $(1-10 \mu \mathrm{M})$ replicated one of the effects of 5-HT, producing significant depression of evoked EPSCs (Fig. 6). The degree of depression produced by 5-CT (mean decrease $66 \pm 8 \%$ ) was the same as that produced by $100 \mu \mathrm{M} 5$-HT (mean decrease $66 \pm 5 \%$ ). However, 5-CT was ineffective in inducing spontaneous IPSCS, producing only a modest increase (31 IPSCs/min) in one neuron of four that were examined. Thus modulation of evoked EPSCs (and possibly of IPSCs as well) is produced at least in part by activation of 5 - $\mathrm{HT}_{1}$ receptors.

In contrast, a 5-HT 2 agonist, $\alpha$-Me-5-HT $(10-100 \mu \mathrm{M})$, reproduced all of the effects of 5-HT. In the cell shown in Fig. $7 A, \alpha$-Me-5-HT induced an increase in spontaneous IPSCs. Similar increases were seen in three of eight cells from P6-8 animals (mean increase $109 \pm 50 \mathrm{IPSCs} / \mathrm{min}$ ); this is comparable with the proportion of cells in which 5-HT induced spontaneous IPSCs. $\alpha$-Me-5-HT also produced significant depression of evoked EPSCs (mean decrease $56 \pm 9 \%$ ) and IPSCs (mean decrease $29 \pm 5 \%$ ) that was similar to the depression produced by $100 \mu \mathrm{M}$ 5-HT (Fig. 7, $B$ and $C$ ).

Next we tested the ability of ketanserin, a $5-\mathrm{HT}_{2}$ antagonist, to block the effects of 5-HT on LSO neurons. In these experiments, 5-HT first was applied in normal ACSF. After washout of 5-HT for $\geq 60 \mathrm{~min}$, ketanserin $(5-20 \mu \mathrm{M})$ was bath applied for $5 \mathrm{~min}$, followed by a second application of 5-HT in the presence of ketanserin. As shown in the example in Fig. 8A, ketanserin blocked the induction of spontaneous IPSCs by 5-HT. In nine such experiments, ketanserin significantly reduced the increase in IPSC frequency produced by 5-HT almost to 0 (Fig. $8 B$ ). However, in the presence of ketanserin, 5-HT still produced significant evoked EPSC depression (Fig. 8, $C$ and $D$ ). Evoked IPSCs were diminished slightly but not significantly.

\section{I S C U S S I O N}

The present study is the first investigation of the effects of 5-HT on the synaptic inputs to LSO. Using whole cell patchclamp recording in brain stem slices from P6 to 13 gerbils, we have demonstrated that 5-HT produces three modulatory actions in LSO neurons: an increase in the rate of spontaneous IPSCs in young $(<\mathrm{P} 9)$ animals, substantial depression of ipsilaterally evoked EPSCs, and modest depression of MNTBevoked IPSCs. At least two 5-HT receptor subtypes are involved in the modulation of LSO synapses. The induction of spontaneous IPSCs was produced by a $5-\mathrm{HT}_{2}$ receptor agonist. Depression of evoked EPSCs was produced by either 5- $\mathrm{HT}_{1}$ or 5-HT ${ }_{2}$ agonists. Depression of evoked IPSCs required a higher concentration of 5-HT $(100 \mu \mathrm{M})$ compared with depression of EPSCs $(10 \mu \mathrm{M})$, implying that different receptors are involved. Similarly, a 5- $\mathrm{HT}_{2}$ agonist reduced evoked IPSCs and the $5-\mathrm{HT}_{2}$ antagonist partially blocked the effect of 5-HT. The potential significance and possible mechanisms of these forms of modulation are discussed in the following text.

Immunohistochemical analysis has revealed that auditory nuclei of the brain stem and midbrain receive serotonergic innervation. Specifically, the cochlear nucleus in rat, guinea pig, and cat exhibits serotonergic immunoreactivity (Klepper and Herbert 1991; Thompson et al. 1994, 1995). In adult animals, serotonergic staining also is found in MNTB but is very sparse or absent in LSO (Thompson et al. 1995). Additionally, $5-\mathrm{HT}_{1 \mathrm{~A}}$ receptors are found in the AVCN (Thompson 
A1

A2
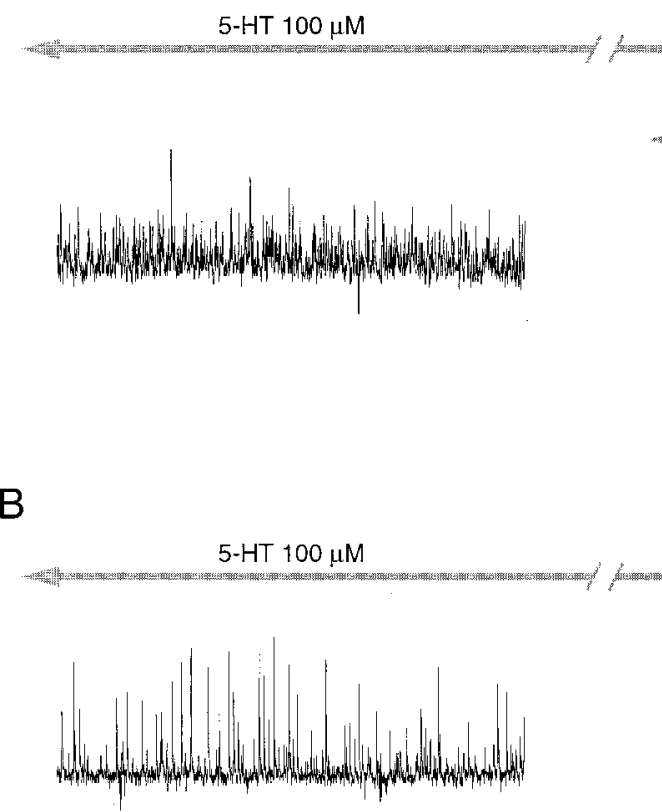

C

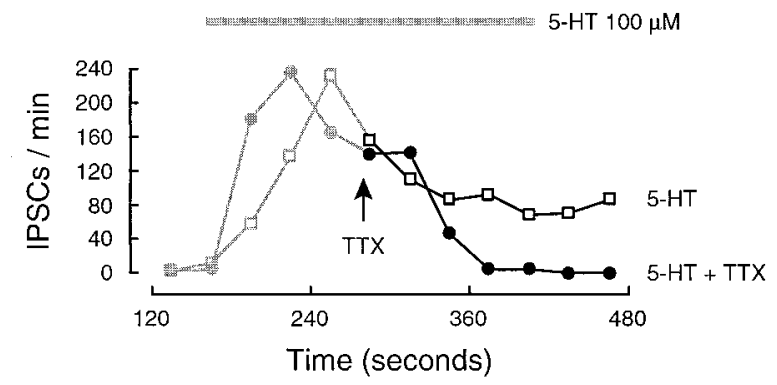

FIG. 3. Blockade of spontaneous IPSCs by tetrodotoxin. Voltage-clamp recordings from a P7 LSO neuron. $V_{\mathrm{H}}=0 \mathrm{mV}$. No spontaneous IPSCs were seen before 5-HT (not shown). Al: $60 \mathrm{~s}$ after 5-HT application, frequent spontaneous IPSCs were evident. TTX $(1 \mu \mathrm{M})$ subsequently was applied. A2: spontaneous IPSCs were abolished $60 \mathrm{~s}$ after TTX application. Elapsed time between the traces is $90 \mathrm{~s} . B$ : after prolonged washout of TTX, 5-HT was reapplied while recording from the same cell, producing persistent induction of IPSCs. Traces correspond to the same time periods after 5-HT application as in $A$. C: IPSC rates from the same experiments are plotted as a function of time. TTX blocked spontaneous IPSCs $(\bullet)$, whereas in the subsequent control experiment $(\square)$, spontaneous IPSCs persisted until the end of the recording period. et al. 1994). Thus both of the auditory nuclei that supply major afferent input to LSO are innervated by $5-\mathrm{HT}$. The serotonergic innervation of these auditory nuclei implies that 5-HT may exert important modulatory influences. In fact, in vivo studies show a predominantly inhibitory action of 5-HT on spontaneous and tone-evoked responses in the cochlear nucleus and inferior colliculus (Ebert and Ostwald 1992; Faingold et al. 1991). However, in vitro intracellular experiments show that 5-HT produces mixed, but primarily excitatory, effects in the ventral nucleus of the trapezoid body and rostral periolivary regions (Wang and Robertson 1997).

\section{Induction of spontaneous IPSCs}

The mechanism by which 5 -HT induces spontaneous IPSCs is not known. Although this effect of 5-HT produces a net increase in inhibition of LSO neurons, the spontaneous IPSCs presumably result from an excitatory action of 5-HT, such as the induction of spontaneous action potential firing in inhibitory neurons that project to LSO. Consistent with this hypothesis, induction of spontaneous IPSCs was blocked by TTX, indicating dependence on $\mathrm{Na}^{+}$action potentials. The major source of inhibitory input to LSO is MNTB, and thus a plausible explanation for our results is that $5-\mathrm{HT}_{2}$ receptors mediate an excitatory action of 5-HT on MNTB neurons. Because induction of spontaneous IPSCs was observed while glutamatergic transmission was blocked, it is likely that 5-HT exerts excitatory actions directly on MNTB neurons rather than

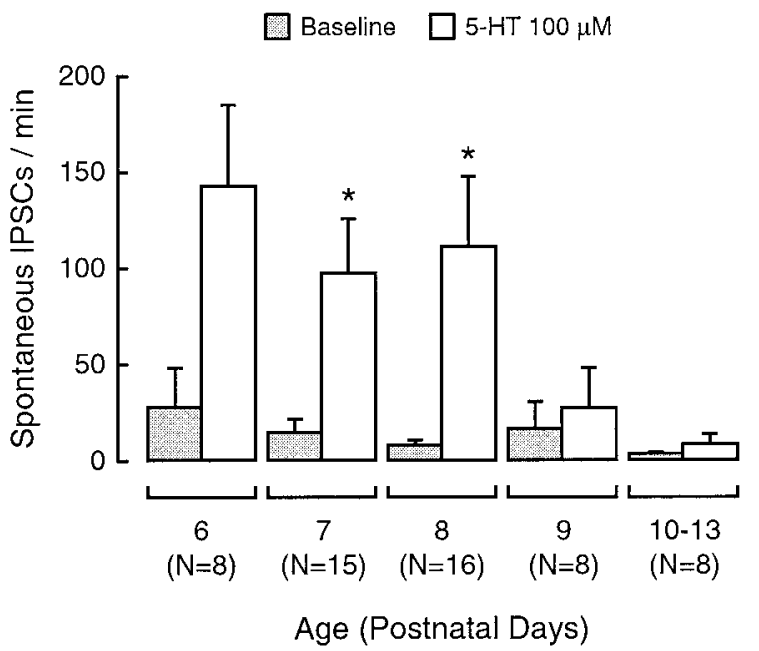

FIG. 4. Induction of IPSCs by 5-HT decreases with postnatal age. Bars indicate the mean rate of spontaneous IPSCs before and after 5-HT application $(100 \mu \mathrm{M})$. Baseline spontaneous rates (filled bars) did not change substantially over the age range examined. 5-HT produced significant increases in the rate of IPSCs at age P7 and 8 , but not at higher ages $(* P<0.01)$. 
A1

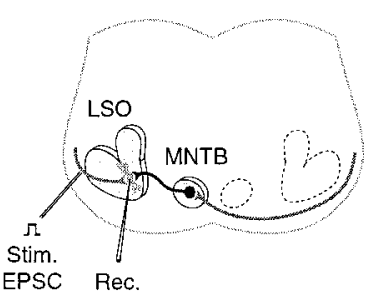

B
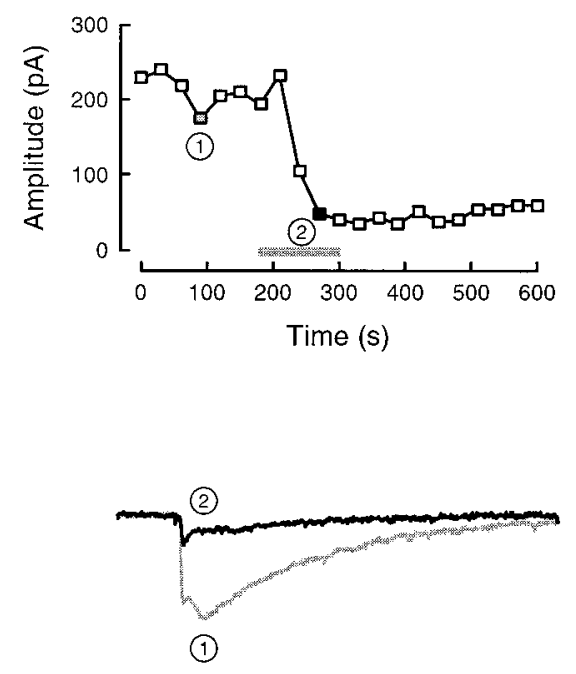

A2

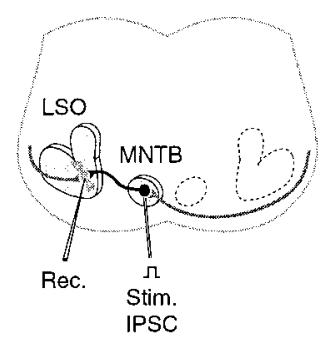

C

Evoked IPSC

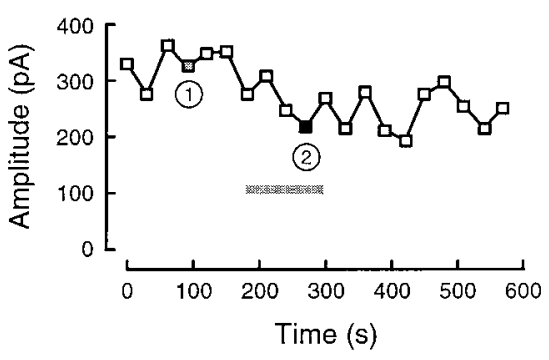

D $\square$ Baseline $\square 5-\mathrm{HT} 10 \mu \mathrm{M}$

E

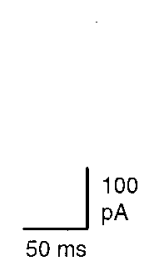

Baseline $\square 5-\mathrm{HT} 100 \mu \mathrm{M}$

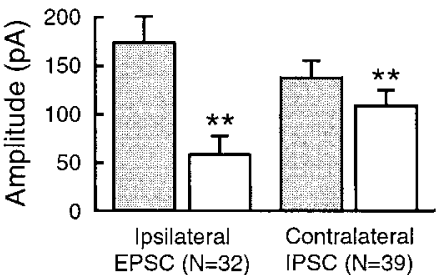

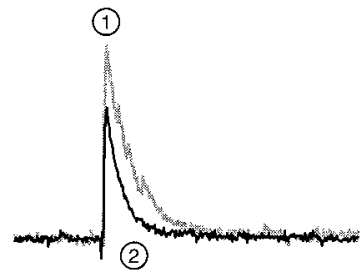

F

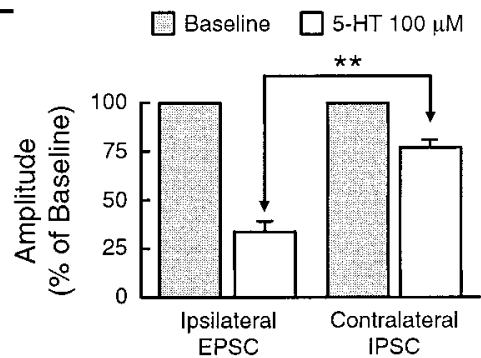

FIG. 5. Modulation of evoked synaptic currents. $A$ : schematic of the stimulation sites used to evoke ipsilateral EPSCs $(A l)$ and contralateral IPSCs (A2) in LSO neurons. $B$ : evoked EPSCs were elicited by afferent stimulation at 30-s intervals. $V_{\mathrm{H}}=-10 \mathrm{mV}$. Filled symbols and numbers correspond to the individual traces shown below. Application of $100 \mu \mathrm{M}$ 5-HT (bar) produced substantial depression of EPSC amplitude. EPSC did not recover from depression within the recording period. $C$ : depression of IPSCs was more modest. $V_{\mathrm{H}}=-20 \mathrm{mV}$. $D: 5$ - HT $(10 \mu \mathrm{M})$ produced significant depression of EPSCs $(* P<0.001)$ but did not affect IPSCs. $E$ : higher concentration of 5-HT $(100 \mu \mathrm{M})$ produced substantial EPSC depression and modest depression of IPSCs (** $P<0.0001)$. $F$ : for comparison purposes, the data from $E$ are expressed as a percentage of baseline amplitude. Serotonergic depression of EPSCs was proportionally greater than that of IPSCs.

polysynaptically. Serotonergic induction of spontaneous IPSPS also has been reported in pyriform cortex and hippocampus; in these areas, 5-HT (acting via 5- $\mathrm{HT}_{2}$ receptors) induces spontaneous firing of inhibitory interneurons (Piguet and Galvan 1994; Segal 1990; Sheldon and Aghajanian 1990). Interestingly, this action of 5-HT in rat hippocampal CA1 neurons is age dependent, occurring only at ages P7-14 (Segal 1990). Serotonergic amplification of spontaneous inhibitory transmission thus may serve a developmental role in several brain systems.

There are several means by which 5-HT could excite MNTB neurons, such as reduction of a tonic hyperpolarizing or shunt- ing membrane current or enhancement of a depolarizing current. One possible mechanism is modulation of the hyperpolarization-activated cationic current, $I_{\mathrm{h}}$, which is prominent in MNTB neurons (Banks et al. 1993). In several cell types, $I_{\mathrm{h}}$ is enhanced by neuromodulators, including 5-HT, resulting in membrane depolarization (Banks et al. 1993; Bobker and Williams 1989; Larkman and Kelly 1992; McCormick and Pape 1990; Takahashi and Berger 1990). Intracellular analysis of MNTB neurons from developing animals will be necessary to elucidate these issues. The induction of spontaneous IPSCs was variable, with no effect occurring in about half of the cells. This variability may indicate that only a subset of MNTB 
A

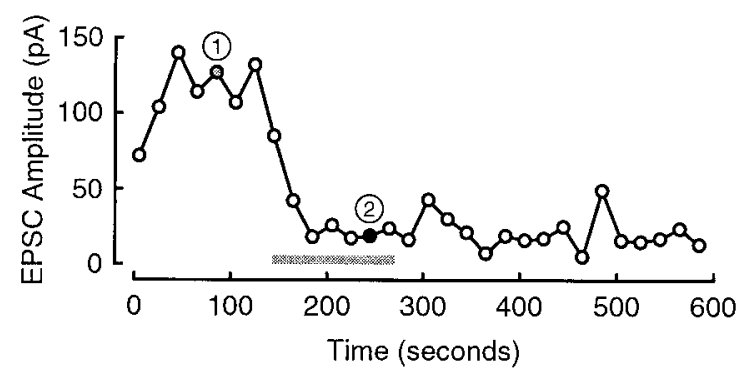

B

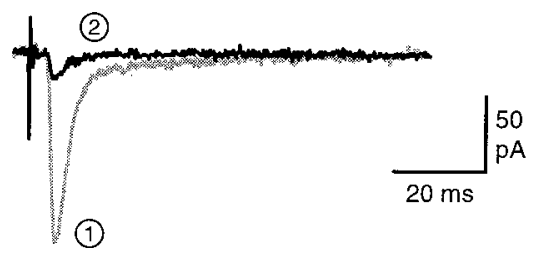

C

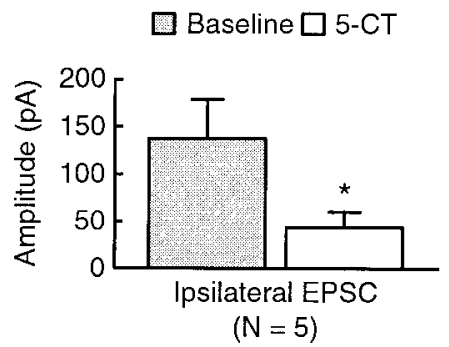

FIG. 6. 5-CT depresses evoked EPSCs. A: 5-CT, a 5-HT 1 agonist $(10 \mu \mathrm{M}$; bar), produced depression of evoked EPSCs in an LSO neuron. $V_{\mathrm{H}}=-20 \mathrm{mV}$. Circled numbers indicate the traces shown in $B . B$ : individual EPSCs measured before (1) and after (2) 5-CT application. $C$ : summary of 5 experiments. 5-CT $(1-10 \mu \mathrm{M})$ produced significant depression of evoked EPSCs $(* P<0.05)$.

neurons are excited by 5-HT; thus not all LSO neurons receive 5-HT-induced IPSCs. Alternatively, induction of IPSCs may require an intact pathway that was disrupted in some brain slices.

Induction of spontaneous IPSCs by 5-HT occurred predominantly in young $(<\mathrm{P} 9)$ animals, raising the possibility that it may serve a developmental role by amplifying inhibitory transmission, which previously has been shown to influence the maturation of LSO structure and function. Such gain may be necessary if spontaneous transmission is to have an influence because spontaneous discharge rates are extremely low during development (Kotak and Sanes 1995). In the first postnatal week, either contralateral cochlear ablation (which removes the excitatory inputs to MNTB) or blockade of glycinergic transmission by systemic strychnine application prevents the normal developmental refinement of MNTB terminal arborizations and LSO dendrites (Aponte et al. 1996; Sanes and Chokshi 1992; Sanes and Hafidi 1996; Sanes and Takacs 1993; Sanes et al. 1992a). These manipulations also influence synaptic function: IPSPs evoked by MNTB stimulation are substantially reduced, and the duration of ipsilateral excitatory postsynaptic potentials is strikingly prolonged (Kotak and Sanes 1995).

Our results suggest that 5-HT could enhance spontaneous activity of MNTB neurons in the first postnatal week, thereby providing appropriate levels of inhibitory synaptic transmission in this period of use-dependent synaptic plasticity. A similar developmental mechanism has been reported in the visual system: spontaneous patterned activity in the retina, mediated by cholinergic transmission, is involved in the activity-dependent plasticity of retinogeniculate afferents before the onset of visual input (Feller et al. 1996; Penn et al. 1998). The cochlea of chick also exhibits patterned activity (Lippe 1994), raising the interesting questions of whether specific patterns of activity, rather than overall activity levels, are important for auditory development and whether neuromodulation contributes to the generation of such patterns.

A final question concerning induction of spontaneous IPSCs is what causes this form of modulation to cease after age P9. Because this action is produced by $5-\mathrm{HT}_{2}$ receptors, one possibility is that the expression of $5-\mathrm{HT}_{2}$ receptors in MNTB decreases with age. Levels of $5-\mathrm{HT}_{2}$ receptors in mouse and rat brain reach a peak at $\sim \mathrm{P} 12-15$ before declining to adult levels (Jazrawi and Horton 1989; Roth et al. 1991). Similar downregulation of 5- $\mathrm{HT}_{2}$ receptors in the superior olivary complex could account for the present findings. Alternatively changes may occur in the second-messenger pathways linked to these receptors or in the membrane currents modulated by $5-\mathrm{HT}$.

\section{Depression of evoked synaptic currents}

We also have found that 5-HT depressed ipsilaterally evoked EPSCs and MNTB-evoked IPSCs recorded in LSO. Because

A

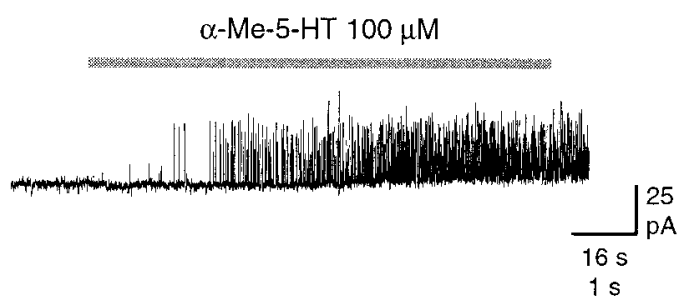

B

EPSC

IPSC
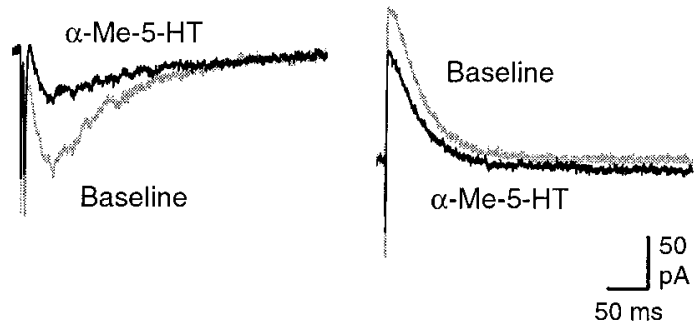

C

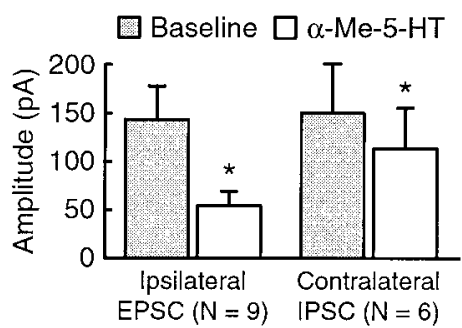

FIG. 7. $\alpha$-Me-5-HT mimics both effects of 5-HT. $A: \alpha-\mathrm{Me}-5-\mathrm{HT}$, a 5-HT 2 agonist, induced spontaneous IPSCs in a P8 LSO neuron. $V_{\mathrm{H}}=-20 \mathrm{mV}$. Evoked EPSCs and IPSCs have been deleted from the trace for clarity. Increases in spontaneous IPSCS were seen in 7 of 9 cells tested. $B$ : like 5-HT, $\alpha$-Me-5-HT preferentially depressed evoked EPSCs. $C$ : summary data. $\alpha$-Me5 -HT produced significant reductions in both evoked EPSCs and IPSCs ( $* P<$ $0.02)$. 
Spontaneous PSCs

A1 Normal ACSF

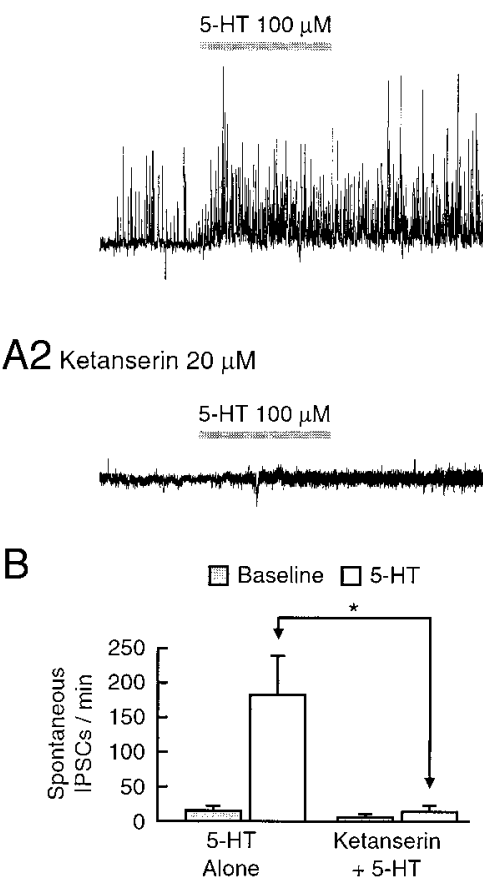

Evoked PSCs

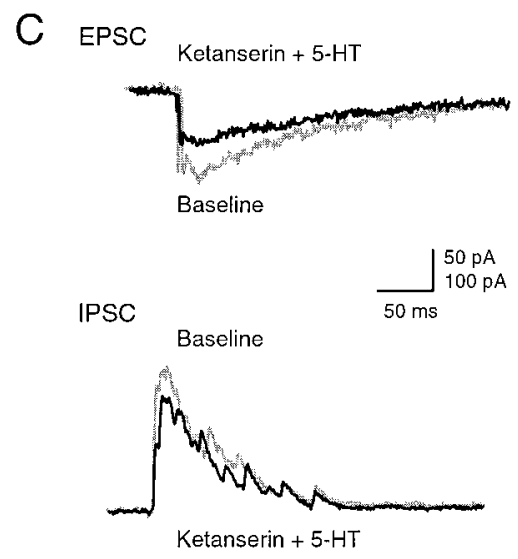

D

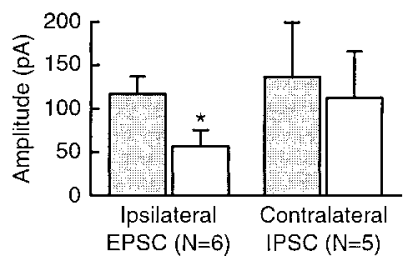

FIG. 8. Ketanserin, a 5-HT 2 antagonist, blocks the induction of spontaneous IPSCs by 5-HT. A1: induction of spontaneous IPSCs by 5-HT in a P6 LSO neuron. $V_{\mathrm{H}}$ $=0 \mathrm{mV}$. A2: after pretreatment with ketanserin $(20 \mu \mathrm{M})$, 5-HT no longer produced spontaneous IPSCs in the same cell. $B$ : summary data from 9 experiments. Ketanserin significantly reduced the number of spontaneous IPSCs induced by 5 -HT $(* P<0.02)$. Baseline spontaneous rates (므) were not affected. $C$ : modulation of evoked EPSCs by 5-HT persisted in the presence of ketanserin. $D$ : summary data. In the presence of ketanserin, 5-HT produced significant depression of EPSC amplitude $(* P<0.05)$. these experiments were performed using voltage clamp with a Cs-containing pipette solution, thereby minimizing the possible contribution of postsynaptic $\mathrm{K}^{+}$conductance, it is likely that the observed depression results either from decreased presynaptic transmitter release or, alternatively, from modulation of postsynaptic receptors. Inhibition of transmitter release by 5 -HT has been described in a number of brain regions. In serotonergic raphe neurons, $5-\mathrm{HT}_{1 \mathrm{~A}}$ receptors produce inhibition resulting from an increase in outward $\mathrm{K}^{+}$current (Aghajanian and Lakoski 1984; Aghajanian and Vandermaelen 1982; Williams et al. 1988). Similar 5-HT $1 \mathrm{~A}^{-}$-mediated inhibition is observed in other brain areas, including cerebral cortex and hippocampus (Bloom et al. 1972; Colino and Halliwell 1987; Segal 1980; Wu et al. 1988). 5-HT produces presynaptic inhibition of synaptic transmission in the hamster superior colliculus via 5-HT 1 r receptors (Mooney et al. 1994). Serotonergic modulation of inhibitory synapses has been reported as well. For example, 5-HT produces presynaptic inhibition of inhibitory interneurons in rat spinal motor nuclei (Umemiya and Berger 1995). It is likely that 5-HT produces presynaptic inhibition of AVCN and MNTB neurons by mechanisms similar to those described in other brain areas. Serotonergic depression of evoked synaptic currents in LSO was reproduced by agonists of either $5-\mathrm{HT}_{1}$ or $5-\mathrm{HT}_{2}$ receptors. It is not yet clear whether these different agonists engage independent mechanisms of depression that produce similar effects or whether both receptors converge on a common pathway.

As described in the previous section, 5-HT frequently induced spontaneous IPSCs in P6-8 LSO neurons. Because both evoked and spontaneous IPSCs may be produced by the same MNTB presynaptic elements, it is possible that the depression of evoked transmission at age P6-8 is due in part to homo- synaptic depression produced by sustained presynaptic action potential firing. However, there was no correlation between the increase in frequency of spontaneous IPSCs and the degree of depression of evoked IPSCs, suggesting that any contribution of homosynaptic depression is relatively minor.

From a functional perspective, our results imply that serotonergic modulation of synaptic transmission in LSO could alter ILD sensitivity in LSO as well as in higher auditory areas that receive input from LSO. Specifically, differential depression of excitatory and inhibitory synapses by 5 -HT would be predicted to diminish the overall responsiveness to acoustic stimuli, modify ILD tuning to favor contralateral inhibitory input, and reduce the slope of ILD tuning functions. In contrast to the age-dependent effect of 5-HT on spontaneous IPSCs, serotonergic modulation of evoked PSCs was seen at all ages examined (P6-13). Although modulation of evoked PSCs has not been tested in adult animals, our results indicate that modulation is present at an age (P12) when the gerbil auditory system is responsive to airborne sound. At this age, LSO neurons exhibit ILD sensitivity, although their response properties are not fully mature: response levels are lower and the slopes of ILD functions are flatter than in adults (Sanes and Rubel 1988). Serotonergic modulation of synaptic transmission in LSO may be a transient effect that persists after P13 but disappears at a later time, raising the possibility that the immature ILD coding properties of LSO neurons may be due in part to the influence of serotonergic modulation. Alternatively, serotonergic modulation of evoked PSCs may persist into adulthood. If this is the case, it will be of great interest to explore the effects of 5-HT on binaural response properties in the mature brain and to determine their functional consequences by using in vivo recording combined with manipula- 
tion of 5-HT levels, either systemically by 5-HT-depleting drugs or 5-HT receptor antagonists, or by local microinjection of serotonergic agonists or antagonists.

We thank two anonymous reviewers for comments on an earlier draft of this manuscript.

This work was supported by National Institute of Deafness and Other Communications Disorders Grants F32 DC-00286 to K. K. Fitzgerald and DC-00540 to D. H. Sanes.

Address for reprint requests: D. H. Sanes, Center for Neural Science, New York University, 4 Washington Place, Room 809, New York, NY 10003.

Received 29 April 1998; accepted in final form 26 February 1999.

\section{REFERENCES}

Aghajanian, G. K. And LaKoski, J. M. Hyperpolarization of serotonergic neurons by serotonin and LSD: studies in brain slices showing increased $\mathrm{K}^{+}$ conductance. Brain Res. 305: 181-185, 1984.

Aghajanian, G. K. And VANDERmaelen, C. P. Intracellular recordings from serotonergic dorsal raphe neurons: pacemaker potentials and the effect of LSD. Brain Res. 238: 463-469, 1982.

Aponte, J. E., KotaK, V. C., and Sanes, D. H. Decreased synaptic inhibition leads to dendritic hypertrophy prior to the onset of hearing. Aud. Neurosci. 2: 235-240, 1996.

Banks, M. I., Pearce, R. A., And Smith, P. H. Hyperpolarization-activated cation current $\left(I_{\mathrm{h}}\right)$ in neurons of the medial nucleus of the trapezoid body: voltage-clamp analysis and enhancement by norepinephrine and cAMP suggest a modulatory mechanism in the auditory brain stem. J. Neurophysiol. 70: 1420-1432, 1993

BeAR, M. F. AND Singer, W. Modulation of visual cortical plasticity by acetylcholine and noradrenaline. Nature 320: 172-176, 1986.

Bennett-Clarke, C. A., Leslie, M. J., Lane, R. D., and Rhoades, R. W. Effect of serotonin depletion on vibrissa-related patterns of thalamic afferents in the rat's somatosensory cortex. J. Neurosci. 14: 7594-7607, 1994.

Bloom, F. E., Hoffer, B. J., Siggins, G. R., Barker, J. L., AND Nicoll, R. A. Effects of serotonin on central neurons: microiontophoretic administration. Fed. Proc. 31: 97-106, 1972.

BOBKER, D. H. AND Williams, J. T. Serotonin augments the cationic current $\mathrm{I}_{\mathrm{h}}$ in central neurons. Neuron 2: 1535-1540, 1989.

Boudreau, J. C. AND Tsuchitani, C. Cat superior olive s-segment cell discharge to tonal stimulation. In: Contributions to Sensory Physiology, edited by W. D. Neff. New York: Academic, 1970, vol. 4, p. 143-213.

CAIRD, D. AND KLINKE, R. Processing of binaural stimuli by cat superior olivary complex neurons. Exp. Brain Res. 52: 385-399, 1983.

CANT, N. B. AND CASSEDAY, J. H. Projections from the anteroventral cochlear nucleus to the lateral and medial superior olivary nuclei. J. Comp. Neurol. 247: 457-476, 1986

Colino, A. And Halliwell, J. V. Differential modulation of three separate K-conductances in hippocampal CA1 neurons by serotonin. Nature 327: 73-77, 1987.

EBERT, U. AND Ostwald, J. Serotonin modulates auditory information processing in the cochlear nucleus of the rat. Neurosci. Lett. 145: 51-54, 1992.

Faingold, C. L., Gehlbach, G., And Caspary, D. M. Functional pharmacology of inferior colliculus neurons. In: Neurobiology of Hearing: The Central Auditory System, edited by R. A. Altschuler, R. P. Bobbin, B. M. Clopton, and D. W. Hoffman. New York: Raven, 1991, p. 223-251.

Feller, M. B., Wellis, D. P., Stellwagen, D., Werblin, F. S., and Shatz, C. J. Requirement for cholinergic synaptic transmission in the propagation of spontaneous retinal waves. Science 272: 1182-1187, 1996.

FitzGerald, K. K. AND SANES, D. H. Serotonergic modulation of inhibitory and excitatory synapses in gerbil lateral superior olive. Soc. Neurosci. Abstr. 22: 649, 1996.

FItZGERALD, K. K. AND SANES, D. H. Serotonergic modulation in gerbil lateral superior olive: effects of 5-HT agonists. Soc. Neurosci. Abstr. 23: 1550, 1997.

Galambos, R., Schwartzkopf, J., and Rupert, A. Microelectrode study of superior olivary nuclei. Am. J. Physiol. 197: 527-536, 1959.

Glendenning, K. K., Hutson, K. A., Nudo, R. J., and Masterton, R. B. Acoustic chiasm. II. Anatomical basis of binaurality in lateral superior olive of cat. J. Comp. Neurol. 232: 261-285, 1985.

Gu, Q. AND SinGER, W. Involvement of serotonin in developmental plasticity of kitten visual cortex. Eur. J. Neurosci. 7: 1146-1153, 1995.
Harvey, J. A., McMaster, S. E., And Romano, A. G. Methylenedioxyamphetamine: neurotoxic effects on serotonergic projections to brainstem nuclei in the rat. Brain Res. 619: 1-14, 1993.

JAZRAWI, S. P. AND HORTON, R. W. 5-HT 2 receptor binding and 5-HT uptake in mouse brain: developmental changes and the relationship to audiogenic seizure susceptibility in DBA/2J mice. Dev. Brain Res. 45: 257-263, 1989.

Kasamatsu, T. and Pettigrew, J. D. Depletion of brain catecholamines: failure of ocular dominance shift after monocular occlusion in kittens. Science 194: 206-209, 1976.

KLEPPER, A. AND HeRBERT, H. Distribution and origin of noradrenergic and serotonergic fibers in the cochlear nucleus and inferior colliculus of the rat. Brain Res. 557: 190-201, 1991.

Kotak, V. C., Korada, S, Schwartz, I. R., and Sanes, D. H. A developmental shift from GABAergic to glycinergic transmission in the central auditory system. J. Neurosci. 18: 4646-4655.

KotaK, V. C. And SANES, D. H. Synaptically evoked prolonged depolarizations in the developing auditory system. J. Neurophysiol. 74: 1611-1620, 1995.

Kotak, V. C. AND SANes, D. H. Developmental influence of glycinergic inhibition: regulation of NMDA-mediated EPSPs. J. Neurosci. 16: 18361843,1996

LaRkman, P. M. and Kelly, J. S. Ionic mechanisms mediating 5-hydroxytryptamine- and noradrenaline-evoked depolarization of adult rat facial motoneurones. J. Physiol. (Lond.) 456: 473-490, 1992.

LAUdER, J. Neurotransmitters as morphogens. Prog. Brain Res. 73: 365-387, 1988.

LIPPE, W. R. Rhythmic spontaneous activity in the developing avian auditory system. J. Neurosci. 14: 1486-1495, 1994.

MCCoRmick, D. A. AND PAPE, H. C. Noradrenergic and serotonergic modulation of a hyperpolarization-activated cation current in thalamic relay neurones. J. Physiol. (Lond.) 431: 319-342, 1990.

Mooney, R. D., ShI, M. Y., AND RhoAdes, R. W. Modulation of retinotectal transmission by presynaptic 5-HT ${ }_{1 \mathrm{~B}}$ receptors in the superior colliculus of the adult hamster. J. Neurophysiol. 72: 3-13, 1994.

Moore, M. J. and Caspary, D. M. Strychnine blocks binaural inhibition in lateral superior olivary neurons. J. Neurosci. 3: 237-247, 1983.

Penn, A. A., Riquelme, P. A., Feller, M. B., And Shatz, C. J. Competition in retinogeniculate patterning driven by spontaneous activity. Science 279 : 2108-2112, 1998.

Piguet, P. And Galvan, M. Transient and long-lasting actions of 5-HT on rat dentate gyrus neurons in vivo. J. Physiol. (Lond.) 481: 629-639, 1994.

Rasmussen, G. L. The olivary peduncle and other fiber projections of the superior olivary complex. J. Comp. Neurol. 84: 141-219, 1946.

Roth, B. L., Hamblin, M. W., and Ciaranello, R. D. Developmental regulation of 5- $\mathrm{HT}_{2}$ and 5-HT $\mathrm{H}_{1 \mathrm{c}}$ mRNA and receptor levels. Dev. Brain Res. 58: $51-58,1991$

SANES, D. H. An in vitro analysis of sound localization mechanisms in the gerbil lateral superior olive. J. Neurosci. 10: 3494-3506, 1990.

SANES, D. H. The development of synaptic function and integration in the central auditory system. J. Neurosci. 13: 2627-2637, 1993.

SANES, D. H. AND CHOKSHI, P. Glycinergic transmission influences the development of dendrite shape. Neuroreport 3: 323-326, 1992.

SANES, D. H. AND HAFIDI, A. Glycinergic transmission regulates dendrite size in organotypic culture. J. Neurobiol. 31: 503-511, 1996.

Sanes, D. H., Markowitz, S., Bernstein, J., AND Wardlow, J. The influence of inhibitory afferents on the development of postsynaptic dendritic arbors. J. Comp. Neurol. 321: 637-644, 1992a.

Sanes, D. H. and Rubel, E. W. The ontogeny of inhibition and excitation in the gerbil lateral superior olive. J. Neurosci. 8: 682-700, 1988.

SANES, D. H. AND SiverLS, V. The development and specificity of inhibitory axonal arborizations in the lateral superior olive. J. Neurobiol. 22: 837-854, 1991.

SANES, D. H., Song, J., AND Tyson, J. Refinement of dendritic arbors along the tonotopic axis of the gerbil lateral superior olive. Dev. Brain Res. 67: 47-55, 1992b.

SANES, D. H. AND TAKaCS, C. Activity-dependent refinement of inhibitory connections. Eur. J. Neurosci. 5: 570-574, 1993.

SEgAL, M. The action of serotonin in the rat hippocampal slice preparation. J. Physiol. (Lond.) 303: 443-456, 1980

SEgAL, M. Developmental changes in serotonin actions in rat hippocampus. Dev. Brain Res. 52: 247-252, 1990. 
Sheldon, P. W. And Aghajanian, G. K. Serotonin (5-HT) induces IPSPs in pyramidal cell layers of rat piriform cortex: evidence for the involvement of a 5-HT $-\mathrm{HT}_{2}$-activated interneuron. Brain Res. 506: 62-69, 1990.

SOMmer, I., LingENHÖHL, K., AND FRIAUf, E. Principal cells of the rat medial nucleus of the trapezoid body: an intracellular in vivo study of their physiology and morphology. Exp. Brain Res. 95: 223-239, 1993.

Spangler, K. M., WarR, W. B., and Henkel, C. K. The projections of principal cells of the medial nucleus of the trapezoid body in the cat. J. Comp. Neurol. 238: 249-262, 1985.

TAKAHASHI, T. AND BERGER, A. J. Direct excitation of rat spinal motoneurones by serotonin. J. Physiol. (Lond.) 423: 63-76, 1990.

Thompson, A. M., Moore, K. R., And Thompson, G. C. Distribution and origin of serotonergic afferents to guinea pig cochlear nucleus. J. Comp. Neurol. 351: 104-116, 1995.

Thompson, G. C., Thompson, A. M., Garrett, K. M., and Britton, B. H. Serotonin and serotonin receptors in the central auditory system. Otolaryngol. Head Neck Surg. 110: 93-102, 1994.

Umemiya, M. And Berger, A. J. Presynaptic inhibition by serotonin of glycinergic inhibitory synaptic currents in the rat brain stem. J. Neurophysiol. 73: 1192-1200, 1995.
WANG, X. AND RoberTSON, D. Effects of bioamines and peptides on neurones in the ventral nucleus of the trapezoid body and rostral periolivary regions of the rat superior olivary complex: an in vitro investigation. Hear. Res. 106: 20-28, 1997.

WARR, W. B. Parallel ascending pathways from the cochlear nucleus: neuroanatomical evidence of functional specialization. In: Contributions to Sensory Physiology, edited by W. D. Neff. New York, Academic, 1982, vol. 7, p. 1-38.

Whitaker-Azmitia, P. M., Druse, M., Walker, P., Lauder, J. M. Serotonin as a developmental signal. Behav. Brain Res. 73: 19-29, 1996.

Williams, J. T., Colmers, W. F., AND PAN, Z. Z. Voltage- and ligand-activated inwardly rectifying currents in dorsal raphe neurons in vitro. J. Neurosci. 8: 3499-3506, 1988.

Wu, S. H. AND Kelly, J. B. NMDA, non-NMDA and glycine receptors mediate binaural interaction in the lateral superior olive: physiological evidence from mouse brain slice. Neurosci. Lett. 134: 257-260, 1992.

Wu, P. H., GuREVICH, N., AND CARLEN, P. L. Serotonin-1a receptor activation in hippocampal neurons by 8-hydroxy-2-(di-n-propylamino) tetralin, 5-methoxytryptamine and 5-hydroxytryptamine. Neurosci. Lett. 86: 72-76, 1988. 